\title{
Band-Gap Tuning and Linear Magnetoresistance in the Silver Chalcogenides
}

\author{
M. Lee, ${ }^{1}$ T. F. Rosenbaum, ${ }^{1}$ M.-L. Saboungi, ${ }^{2}$ and H. S. Schnyders ${ }^{3}$ \\ ${ }^{1}$ The James Franck Institute and Department of Physics, The University of Chicago, Chicago, Illinois 60637 \\ ${ }^{2}$ Argonne National Laboratory, Argonne, Illinois 60439 \\ and CRMD-CNRS, Orléans, Cedex 2 France \\ ${ }^{3}$ Physics Department, Lake Forest College, Lake Forest, Illinois 60045
}

(Received 25 June 2001; published 24 January 2002)

\begin{abstract}
Optimally doped silver selenide and silver telluride exhibit linear positive magnetoresistance over decades in magnetic field and on a scale comparable to the colossal magnetoresistance compounds. We use hydrostatic pressure to smoothly alter the band structure of Ag-rich and Ag-deficient samples of semiconducting $\mathrm{Ag}_{2 \pm \delta} \mathrm{Te}$ of fixed stoichiometry and disorder. We find that the magnetoresistance spikes and the linear field dependence emerges when the bands cross and the Hall coefficient changes sign.
\end{abstract}

DOI: $10.1103 /$ PhysRevLett.88.066602

The magnetoresistive response of a material can open a window into the dispersion and dynamics of the charge carriers, and in opportune cases can be exploited for technological use. The magnetoresistance at small fields is usually quadratic because of the vector nature of the magnetic field, and is expected to saturate when the applied field becomes large [1]. Under special circumstances, the resistivity can grow linearly with applied field [2]. High-field linear magnetoresistance can be found in polycrystalline materials with open orbits in the Fermi surface [3], in inhomogeneous materials where the tensor components of the resistivity can be mixed [4], and in the extreme quantum limit where one Landau level dominates (e.g., bismuth) [5-7].

A few years ago, positive linear magnetoresistance was observed from magnetic fields of $\mathrm{mT}$ to $\mathrm{T}$ in the silver chalcogenides, $\mathrm{Ag}_{2} \mathrm{Se}$ and $\mathrm{Ag}_{2} \mathrm{Te}$ [8]. Stoichiometric material remains indifferent to the application of a magnetic field [9], but small amounts of excess silver or excess $\mathrm{Se} / \mathrm{Te}$ lead to changes in the resistivity of many hundreds of percent in fields of a few $\mathrm{T}$. This large magnetic response is comparable in absolute magnitude to that observed in manganese perovskites, the so-called colossal magnetoresistance materials, but occurs here in intrinsically nonmagnetic materials that can be fabricated both in bulk and as thin films [8,10-13].

The unusually large range of linearity observed in some samples of $\mathrm{Ag}_{2}+\delta$ Se and $\mathrm{Ag}_{2}+\delta$ Te, the failure of the magnetoresistance to saturate even when the product of the cyclotron frequency and the scattering time, $\omega_{c} \tau$, greatly exceeds one, and the robust absolute scale of the response combine to make the silver chalcogenides especially inviting materials to illuminate the mechanisms of linear magnetoresistance. In particular, Abrikosov has proposed that an essential ingredient for "quantum linear magnetoresistance" in both the small and large field limits is a semiconducting gap that approaches zero, with an energy dispersion that becomes linear in momentum $[2,14]$.

In this Letter, we use hydrostatic pressure to tune the band gap of both $p$-type and $n$-type samples of silver tel-
PACS numbers: 72.20.My, 72.15.Gd, 72.80.Jc

luride of fixed stoichiometry and disorder. Under pressure, hole-dominated transport can be transformed into electron-dominated transport, and barely metallic $n$-type samples can be converted into good metals. We find for $p$-type material that both the linear magnetoresistance and the largest amplitude response emerge precisely where the Hall coefficient crosses through zero. At high pressures, well into the metal, the magnetoresistance is quenched and the expected quadratic dependence on field is recovered.

Appropriately weighted amounts of high purity $\mathrm{Ag}$ (99.999\%, Alfa Aesar) and Te $(99.9999 \%$ pure, metals basis, Alfa Aesar), sealed in quartz tubes under a vacuum better than 5 mtorr, were melted to create polycrystalline samples at desired stoichiometries, both silver rich ( $n$-type) and silver deficient ( $p$-type). The compound was rocked at $50{ }^{\circ} \mathrm{C}$ above the reported melting point to ensure complete mixing. Slowly cooled samples were cut perpendicular to the long axis of the cylindrical boule to avoid dopant variations due to small temperature gradients in the furnace. We applied hydrostatic pressures $P$ up to 17 kbar using a $\mathrm{BeCu}$ piston-anvil self-locking pressure clamp with a tungsten carbide insert. Samples of typical dimensions $(3.0 \times 0.8 \times 0.8) \mathrm{mm}^{3}$, as well as a chip of $\left(\mathrm{V}_{0.99} \mathrm{Ti}_{0.01}\right)_{2} \mathrm{O}_{3}$ for use as a manometer [15], were sealed in the Teflon bucket using Fluorinert as the pressure medium. The pressure cell was then mounted in a helium-3 cryostat centered in an $8 \mathrm{~T}$ superconducting magnet, with the long axis of the sample perpendicular to the magnetic field direction. We performed four-probe resistivity and five-probe Hall coefficient measurements using a conventional ac bridge technique in the Ohmic and frequency-independent limits. The relative error in the resistivity is less than $0.01 \%$, but there is an absolute uncertainty of $20 \%$ because of the finite extent of multiple InBi solder contacts on samples of millimeter dimensions.

We plot in Fig. 1 an overview of the temperature dependence of the longitudinal resistivity of a tellurium-rich sample at a series of hydrostatic pressures $P$. The band structure is altered by $P$ such that a dominantly $p$-type sample (filled symbols) at ambient pressure assumes 


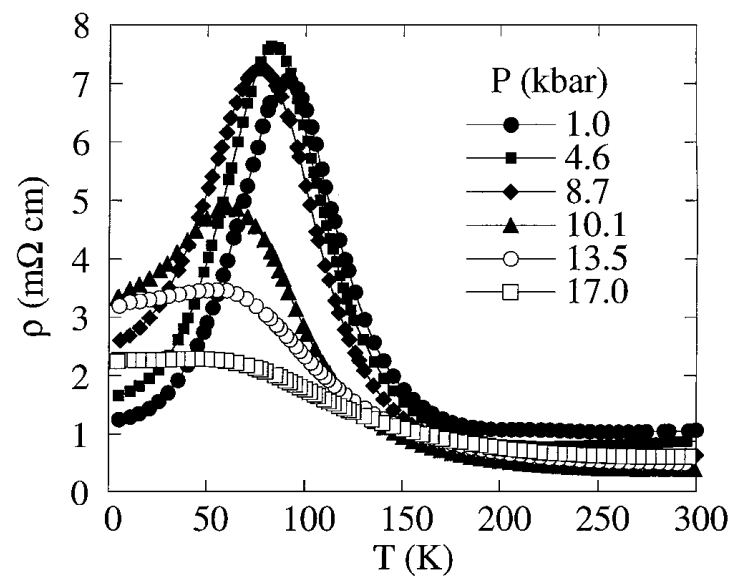

FIG. 1. Longitudinal resistivity $\rho_{x x}$ vs temperature $T$ at a series of hydrostatic pressures $P$ for a sample of $\mathrm{Ag}_{2-\delta} \mathrm{Te}$, a self-doped narrow-gap semiconductor. Solid symbols indicate predominant $p$-type character, crossing over to $n$-type character (open symbols) at high pressure (see Fig. 2b). Solid lines are guides to the eye.

$n$-type character (open symbols) by high pressure. The nonmonotonic $\rho(T)$ reflects the behavior of a narrow-gap self-doped degenerate semiconductor. Between $T=0.4 \mathrm{~K}$ and 60 to $100 \mathrm{~K}$ the resistivity rises because the effective carrier density is constant and the phonon scattering increases with $T$. At higher $T$, the transport becomes activated and $\rho$ decreases with increasing $T$ in accordance with the usual behavior of small-gap semiconductors.

Our key experimental result is contained in the two halves of Fig. 2. As a function of hydrostatic pressure at fixed temperature, the magnetoresistance smoothly evolves from a sublinear magnetic field dependence to a superlinear field dependence. At $P=10.1 \mathrm{kbar}$, the magnetoresistance is linear in $H$ over decades in field. It is also greatly enhanced. The robust linear field dependence emerges hand-in-hand with the maximum magnetoresistive response, which exceeds $200 \%$ by $H=6 \mathrm{~T}$.

The Hall coefficient (bottom half of Fig. 2) traces out the closing of the band gap deep in the intrinsic regime. Hole-dominated transport gives rise to a sublinear $\rho_{x x}(H)$; electron-dominated transport gives rise to a superlinear $\rho_{x x}(H)$. In both regimes, the magnetoresistance remains positive. Remarkably, the point where the magnetoresistance becomes linear in field and attains its maximum value is where $R_{H} \rightarrow 0$ : at the crossover from $p$-type to $n$-type character.

The striking enhancement of the magnetoresistance at $P=10.1$ kbar jumps out from Fig. 2 at the higher magnetic fields. For example, at $H=6 \mathrm{~T}$ it is more than twice the value of the normalized magnetoresistance at $P=8.7$ kbar, and almost thrice the normalized magnetoresistance at $13.5 \mathrm{kbar}$. Less obvious from the figure is the enhancement at small magnetic fields. We limn the intimate connection between the diagonal and off-diagonal components of the resistivity tensor in Fig. 3. Whether
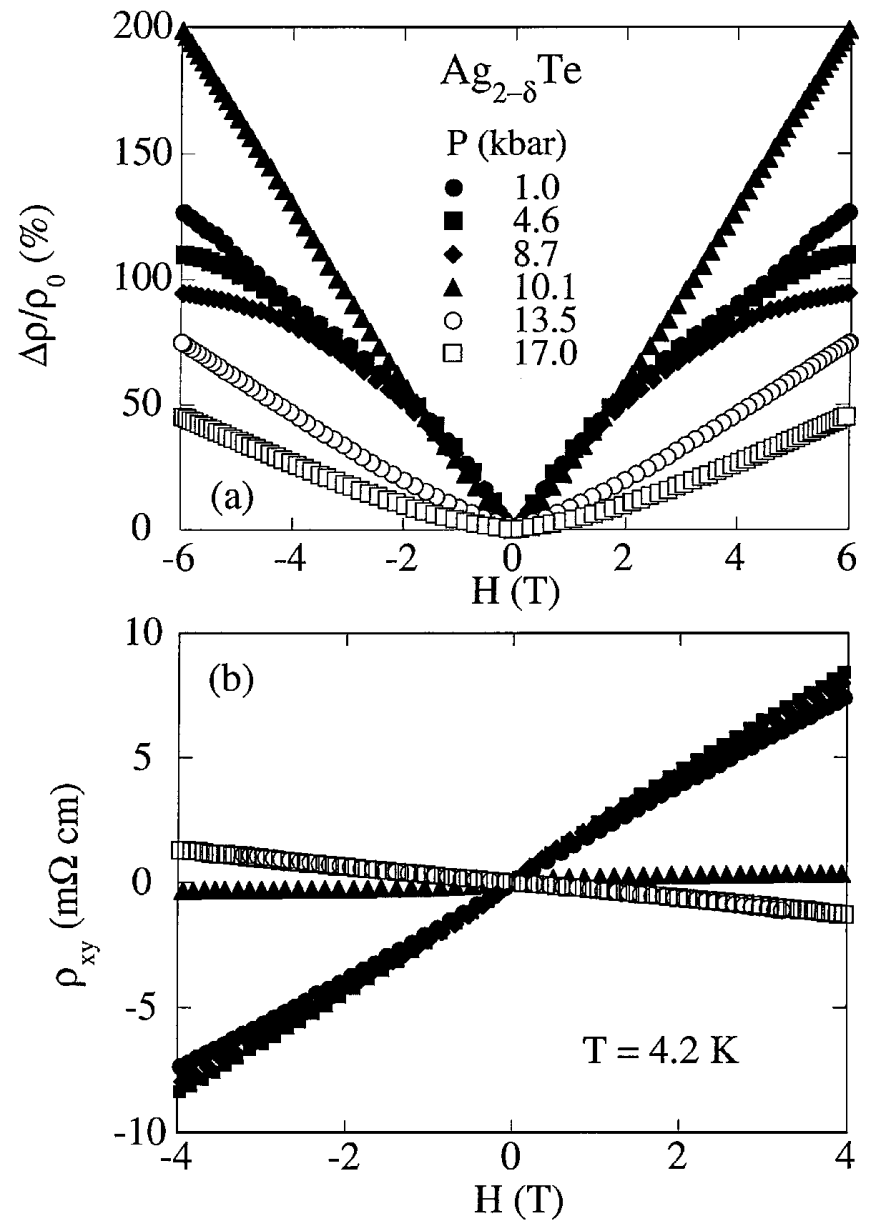

FIG. 2. (a) Normalized magnetoresistance, $[\rho(H)-\rho(0)] /$ $\rho(0)$, and (b) Hall resistivity, $\rho_{x y}$, at $T=4.2 \mathrm{~K}$ as a function of magnetic field $H$ at pressures $P$. Linear magnetoresistance emerges at the point where the Hall resistivity crosses through zero.

in the low-field limit $\left(H=0.1 \mathrm{~T}, \omega_{c} \tau \sim 0.1\right)$ or in the high-field limit $\left(H=5.5 \mathrm{~T}, \omega_{c} \tau \sim 5\right)$, the magnetoresistance peaks when the Hall coefficient changes sign. Echoes of this behavior can be seen in the local maximum of the magnetoresistance as a function of temperature [12]. Such maxima are found near the temperature where the sum of the products of the density and mobility for each type of carrier approaches a minimum. However, the strong nonmonotonic variation of $\rho_{x y}(H)$ for $60<T<130 \mathrm{~K}$ precludes a generally applicable analysis at all $H$, a complication obviated here by fixing $T$ in the low temperature, constant carrier density regime and introducing a new variable, hydrostatic pressure $P$.

Figures 2 and 3 highlight the pivotal role played by band crossing in $p$-type material. When the bands cross and the Hall coefficient changes sign, the magnetoresistance spikes. From Fig. 1, we see that the low temperature resistivity also has a maximum at this point $(P=10.1 \mathrm{kbar})$. Although the effective mass necessarily becomes very small when the band gap closes, the effective carrier 


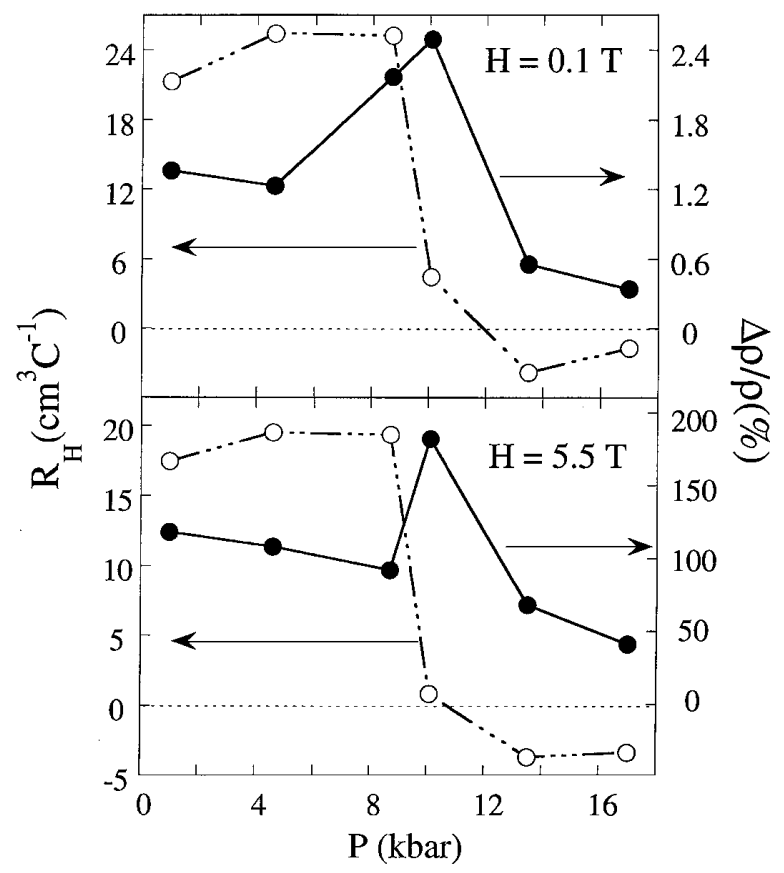

FIG. 3. The intertwined relationship of the Hall coefficient $R_{H}$ (open circles, left axis) and the magnetoresistance, $\Delta \rho / \rho$ (filled circles, right axis), at $T=4.2 \mathrm{~K}$. At both low and high magnetic fields, the normalized magnetoresistance peaks at the pressure where $R_{H}$ changes sign.

density approaches zero as well. Hence, $\rho(P) \propto m^{*}(P) /$ $n_{\text {eff }}(P)$ can reach a maximum rather than a minimum. Pressure experiments on the canonical narrow-gap semiconductor, $\mathrm{Hg}_{1-x} \mathrm{Cd}_{x} \mathrm{Te}$, likewise reveal a resistivity maximum in the gapless state [16].

We complement our studies of silver-deficient $\mathrm{Ag}_{2-\delta} \mathrm{Te}$ with an investigation of the effects of pressure on a silverrich sample of $\mathrm{Ag}_{2+\delta} \mathrm{Te}$. We show in the inset of Fig. 4 the pressure evolution of the Hall resistivity at $T=4.2 \mathrm{~K}$, and in the main figure the corresponding magnetoresistive response. On this side of the divide, $n$-type material just becomes more so, with net carrier density increasing from $1.4 \times 10^{18}$ to $1.8 \times 10^{19} \mathrm{~cm}^{-3}$ as $P$ increases from 0 to 11.7 kbar. Driving the system more metallic quenches the magnetoresistance. A $1 \mathrm{~T}$ field increases the longitudinal resistivity by only $6 \%$ at $P=11.7$ kbar.

Driving the $\mathrm{Ag}_{2} \mathrm{Te}$ system away from the band-crossing region via either band filling [8] or band broadening (Figs. 2 and 4) reduces the magnitude of the magnetoresistance and changes its functional form. We note, however, that $R_{H} \rightarrow 0$ is not a sufficient condition for large and linear magnetoresistance. The Hall coefficient must cross through zero, not simply approach zero at high carrier density. Details of the band structure, or perhaps differences between the distribution profiles of the excess silver and the excess tellurium, lead to asymmetry about $n_{\text {eff }}=0$.

Compressing nonstoichiometric samples of $\mathrm{Ag}_{2 \pm \delta} \mathrm{Te}$ permits us to manipulate the band structure and optimize the response. As predicted by Abrikosov [2,14], when the

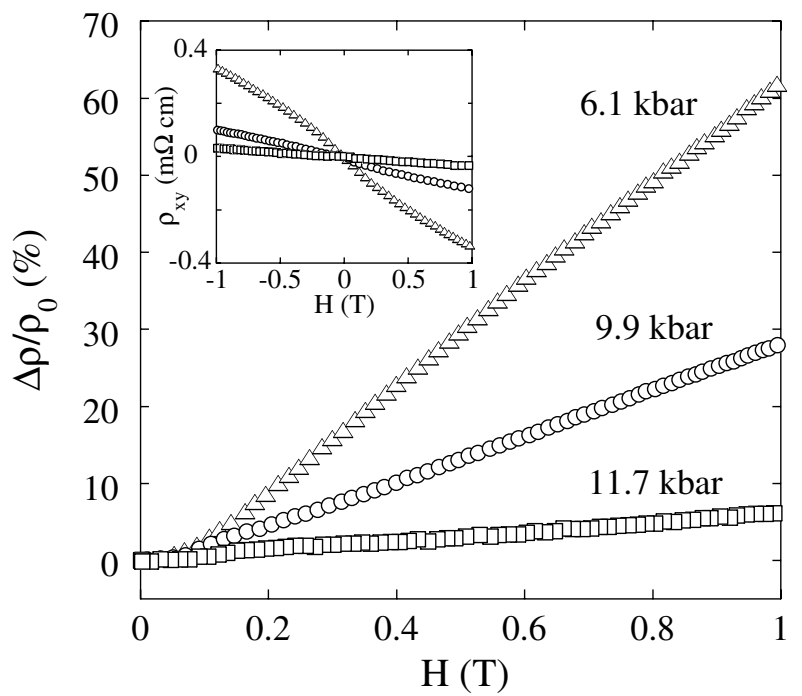

FIG. 4. The magnetoresistance at $T=4.2 \mathrm{~K}$ for a highly doped sample of $\mathrm{Ag}_{2+\delta}$ Te decreases with increasing pressure. The response is field independent at low $H$, with an onset magnetic field $H \sim 0.1 \mathrm{~T}$, from which we ascertain the presence of correlated regions of excess silver. Inset: Hall resistivity at the same pressures, indicating the more metallic nature of the sample with increasing $P$.

bands touch, the linear magnetoresistance emerges. However, even at band crossing, it is important to recognize the fundamental role played by disorder. Experimentally, stoichiometric $\mathrm{Ag}_{2} \mathrm{Te}$ appears uninteresting in the magnetoresistance context [9]. Theoretically, both classical [4] and quantum [14] scenarios require an inhomogeneous medium with highly conducting regions, due, for example, to excess silver, embedded in less conducting material. Fluctuations in the conductivity permit length scales to emerge that are not simply set by the cyclotron radius, and can lead to a linear field dependence of the resistivity over many orders of magnitude in $H$.

If the conductivity fluctuations are sufficiently pronounced leading to connected regions, then the physical mechanism for linear magnetoresistance fades in importance and geometrical effects can dominate. "Designer" sensors, with embedded metal inhomogeneities, exhibit geometrical magnetoresistance of many thousands of percent [17]. When the inhomogeneities are sufficiently large, distorted current paths give rise to onset behavior at finite magnetic field. Highly doped $\mathrm{Ag}_{2+\delta} \mathrm{Te}$, well away from the region where the bands cross and the magnetoresistance spikes, shows traces of such onset behavior. We see in Fig. 4 a field-independent resistivity at each pressure for $H<0.1 \mathrm{~T}$, above which the positive magnetoresistance sets in. An onset $H \sim 0.1 \mathrm{~T}$, corresponding to $\omega_{c} \tau \sim 0.1$, indicates that the excess silver combines to form highly conducting regions of significant spatial extent.

In summary, we use hydrostatic pressure to tune the band structure of both Te-rich and Ag-rich polycrystals 
of $\mathrm{Ag}_{2 \pm \delta}$ Te. Just when the electron and hole bands cross [18], where the Hall coefficient changes sign, the magnetoresistance spikes and becomes linear in field, in accord with Abrikosov's predictions [2,14] for quantum linear magnetoresistance. Results at high pressure establish that although $\delta$ is small $\left(\sim 10^{-4}\right)$, highly conducting regions of significant diameter exist within a more insulating medium.

We are grateful to A. A. Abrikosov, A. Husmann, and P. B. Littlewood for illuminating discussions. The work at the University of Chicago was supported by U.S. Department of Energy Grant No. DE-FG02-99ER45789. The work at ANL was supported by the U.S. Department of Energy, Division of Materials Sciences, Office of Basic Energy Sciences, under Contract No. W-31-109-ENG-38.

[1] See, for example, J.R. Ziman, Principles of the Theory of Solids (Cambridge University Press, Cambridge, 1972), pp. 250-254.

[2] For a cogent exposition of the history and physics of linear magnetoresistance, see A. A. Abrikosov, Europhys. Lett. 49, 789 (2000).

[3] P. L. Kapitza, Proc. R. Soc. London A 123, 292 (1929).
[4] C. Herring, J. Appl. Phys. 31, 1939 (1960); A. C. Beer, in Solid State Physics, Suppl. 4, edited by F. Seitz and D. Turnbull (Academic Press, New York, 1960), p. 104.

[5] P. L. Kapitza, Proc. R. Soc. London A 119, 328 (1928).

[6] F. Y. Yang, K. Liu, D. H. Reich, P. C. Searson, and C. L. Chien, Science 284, 1335 (1999).

[7] A. A. Abrikosov, Sov. Phys. JETP 29, 746 (1969).

[8] R. Xu, A. Husmann, T.F. Rosenbaum, M.-L. Saboungi, J. E. Enderby, and P. B. Littlewood, Nature (London) 390, 57 (1997).

[9] P. Junod, Helv. Phys. Acta 32, 567 (1959).

[10] I. S. Chuprakov and K. H. Dahmen, Appl. Phys. Lett. 72, 2165 (1998).

[11] Z. Ogorelec, A. Hamzic, and M. Basletic, Europhys. Lett. 46, 56 (1999).

[12] H. S. Schnyders, M.-L. Saboungi, and T. F. Rosenbaum, Appl. Phys. Lett. 76, 1710 (2000).

[13] S. S. Manoharan, S. J. Prasanna, D. E. Kiwitz, and C. M. Schneider, Phys. Rev. B 63, 212405 (2001).

[14] A. A. Abrikosov, Phys. Rev. B 58, 2788 (1998).

[15] T. F. Rosenbaum, S. A. Carter, and J. M. Honig, Rev. Sci. Instrum. 67, 617 (1996).

[16] N. B. Brandt, O. N. Belousova, L. A. Bovina, V. I. Stafeev, and Ya. G. Ponomarev, Sov. Phys. JETP 39, 157 (1974).

[17] S. A. Solin, T. Thio, D. R. Hines, and J. J. Heremans, Science 289, 1530 (2000).

[18] S. A. Aliev and F. F. Aliev, Izv. Akad. Nauk SSSR, Neorg. Mater. 21, 1869 (1985) [Inorg. Mater. 21, 1627 (1985)]. 